\title{
ENABLING A TRANSFORMING ARMY AT WAR: ANALYSIS TO IMPROVE LOGISTICS NETWORK EFFICIENCY AND EFFECTIVENESS
}

\author{
Greg Parlier \\ Office of Economic Development \\ University of Alabama at Huntsville \\ Huntsville, AL 35899, U.S.A.
}

\begin{abstract}
This paper introduces and summarizes a comprehensive systems approach guiding an ongoing project addressing these significant challenges confronting logistics transformation. Currently sponsored by the Army Aviation and Missile Command (AMCOM), this project involves several supporting organizations both within and external to the Army and DOD. Although initially focused on aviationspecific Class IX (spare parts and components) as a test bed, the goal is to develop a prototype that will provide the foundational "analytical architecture" to support, guide and accelerate Army Logistics Transformation. Conditions which motivate this research and analysis include (1) the changed nature of our geopolitical landscape resulting in the Army's transition to a "capabilities-based" force, (2) the opportunity to consider, adapt and extend, where appropriate, integrating "supply chain" design, management and analysis concepts that have been driven by increasing competition in the corporate world, and (3) the enabling potential of information technology.
\end{abstract}

\section{INTRODUCTION}

The United States Army, fully engaged in the Global War on Terrorism, is simultaneously committed to the most ambitious and comprehensive "reengineering" endeavor in its history. Universally known by the ubiquitous term "Army Transformation", it has been described as a "continuous process that creates a culture of innovation, which in turn seeks to exploit and shape the changing conduct of military competition." The early intellectual stages of this effort clearly revealed a crucial prerequisite to transform logistics concepts, organization, technology, and culture in order to improve strategic responsiveness, force projection, and sustainment capabilities. Fundamentally, without a transformation in logistics there can be no Army Transformation. Recent operational experience reinforces this assertion and further provides both urgency and a compelling, chronic need for improvement (Association of the United States Army 2004, Kallock and Williams 2004). Although countless "great ideas" and technology initiatives have been offered (Headquarters Department of the Army 2003), the challenge for the analytical community is to recognize and fully comprehend the fundamental nature of this predicament and then to offer an "analytical architecture" that will guide the Army through this transformative period.

This paper introduces and summarizes a comprehensive systems approach guiding an ongoing project addressing these significant challenges confronting logistics transformation. Currently sponsored by the Army Aviation and Missile Command (AMCOM), this project involves several supporting organizations both within and external to the Army and DOD. Although initially focused on aviationspecific Class IX (spare parts and components) as a test bed, the goal is to develop a prototype that will provide the foundational "analytical architecture" to support, guide and accelerate Army Logistics Transformation. Conditions which motivate this research and analysis include (1) the changed nature of our geopolitical landscape resulting in the Army's transition to a "capabilities-based" force, (2) the opportunity to consider, adapt and extend, where appropriate, integrating "supply chain" design, management and analysis concepts that have been driven by increasing competition in the corporate world, and (3) the enabling potential of information technology.

\section{BACKGROUND}

The project this article will address originated in the form of two seemingly simple questions posed by the Commanding General of AMCOM (Parlier 2003). Despite exponential growth in both requirements for, and investment in, aviation spare parts over the preceding five-year period, unfinanced requirements (UFRs) and associated backorders had been growing dramatically. In fact, at the beginning of FY03 the UFR for aviation spares alone was in excess of \$1 Billion. Readiness reports had been slowly declining at the same 
time although not precipitously so. However, there was growing skepticism in the accuracy of these reports and tactical-level "workarounds" in the field were known to be increasing as well. Consequently, it was not clear what impact, in terms of an incremental increase in actual readiness, fully funding the growing spares shortfall might have. Long depot repair and procurement lead times are associated with many of these components, and both obsolescence and diminishing sources of manufacturing supply characterize increasingly aging Army rotorcraft fleets. At the same time, another worrisome pattern seemed to be emerging. Major systems across the Army increasingly were being rated nonoperational due to relatively inexpensive spare parts. The Army was engaged in Operation Enduring Freedom at the time. The growing fear, just a few short months prior to initiating Operation Iraqi Freedom, was that significant additional stress to the aviation fleet could result in sustained readiness deterioration in terms of aircraft operational availability. The combined effect of these trends, at worst, seemed to portray an organization faced with the proverbial "death spiral"- decreasing performance in the face of rapidly escalating costs at a time of potentially devastating consequences. At best, the actual location in a conceptual investment-performance trade space, defined in this case as cost-availability, was uncertain and, relative to an "efficient frontier," simply unknown. (See Figure 1.) The AMCOM CG's "simple" questions, posed back in the Fall, 2002 when these conditions existed, were: "How much should we be investing on spares at the wholesale level to meet fleet readiness goals?" and "Are we spending our resources on the right things?"

\section{Assessment}

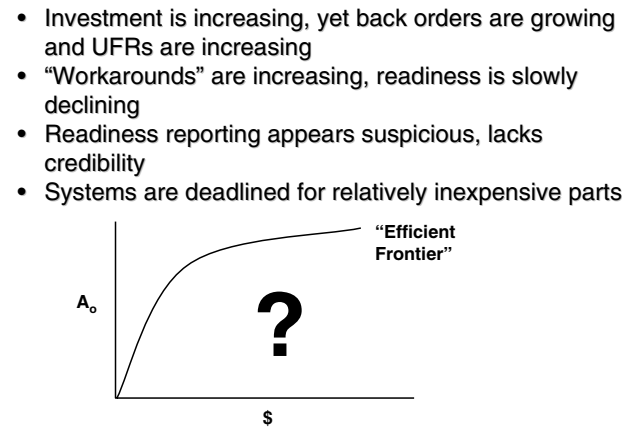

Figure 1: Assessment of Conceptual Investment and Performance

\section{CURRENT LOGISTICS STRUCTURE}

This initial article presents a systems framework, conceptually a multi-stage, logistics model, that is guiding this project. Subsequent articles will explain in greater detail particular stages, emerging analytical insight and supporting recommendations from the various participating analytical organizations. The multi-stage conceptual model is a graphical representation of the logistics structure. (See Figure 2.) It consists of the following "stages": a "unit" stage representing Army tactical organizations where readiness "production" actually occurs; a "demand" stage representing training requirements and operational missions; a "retail" stage representing installation and tactical supply support activities providing direct or general support to specific "units"; a "wholesale" stage consisting of the aggregate CONUS- based repair and supply depots managed by Army Materiel Command and the Defense Logistics Agency; a "reverse logistics" stage representing the retrograde pipeline for depot-level repair components (DLRs), including turbine engines, transmissions, and rotor blades for aviation systems; and an "acquisition" stage representing original equipment manufacturers and suppliers responding to the procurement needs of the Army.

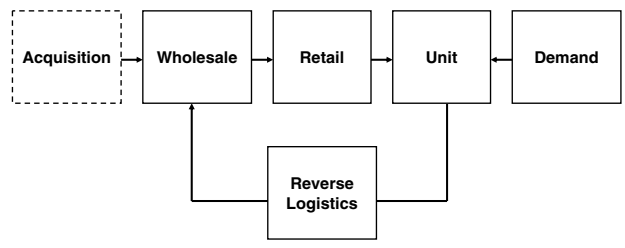

Figure 2: Conceptual Model of Logistics Structure

This multi-stage conceptual perspective, coupled with basic knowledge about the current state of logistics, reveals some initial insight into the potential cause of challenges that have been accumulating over time. For example, unlike the corporate sector where consumer demand is well defined at the end of the supply chain and product consumption can be measured precisely and forecasted accurately, the "customer" here is "readiness" at the unit needed to meet operational mission requirements. A well-defined and understood production function, or "readiness equation," especially for Army aviation systems, relating capital investment and labor to various aircraft performance standards necessary to meet the "demand" for training and mission requirements does not yet exist. Without it, efforts to forecast logistics requirements have been inaccurate and largely reactive leading to uncertainty and variability induced into the larger logistics "system" at the point of consumption.

Another example is the existence of a "reverse logistics" retrograde stage. Rare in the business world since products are normally consumed by the market, this stage constitutes the "value recovery" effort to rebuild, repair and return large subassemblies and replaceable units which are not "consumed" but "used" as capital assets. Although 
these items constitute only about $25 \%$ of demand, they also represent more than $75 \%$ of value. Nonetheless, an underlying theory for retrograde operations has never been established to guide performance standards for the reverse pipeline and, as a consequence, the organizations responsible for the forward supply chain are also responsible for the reverse. Until recently, a capability to measure the delay in the reverse pipeline did not even exist reflecting the lack of importance and priority this aspect of logistics operations historically received within the culture. Yet, this multi-stage conceptual model reveals the importance of viewing the retrograde stage, from a systems control theory perspective, as a "feedback loop" with obvious impact upon "output" generated as unit readiness. For every repairable item delayed in an unresponsive reverse stage, and delay times are now measured in averages of several months, another like component must exist elsewhere within the system or the "feedback loop" will degrade "output."

Finally, the multi-stage model also suggests the current logistics structure is configured as a series of independently operating organizations, frequently with differing agendas and conflicting goals, managing the adjacent interfaces between them. (See Figure 3.) Not surprisingly, over the years various performance metrics evolved to manage these interfaces. However, these organizations did not have good visibility of their effect upon "readiness" production and could not relate their interface metrics to a readinessoriented outcome. Consequently, an ability to effectively correlate resource investment levels and coordinate policy decisions-both within the "stages" and across them-to achieve readiness goals could not be achieved. In short, the existing logistics structure, largely a "legacy" system from an industrial age environment where "buffers" of stock were created to accommodate uncertainty and variability, was never "designed" to answer the AMCOM CG's questions.

\section{Improving System Effectiveness: Integration and Optimization}

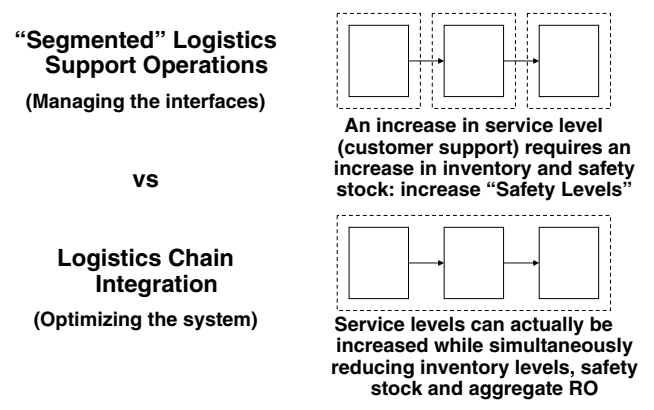

Figure 3: Logistics Structures

\section{ANALYTICAL FOUNDATION FOR IMPROVING SYSTEM EFFECTIVENESS}

The academic development of theory and subsequent practical implementation of "supply chain" management concepts in the corporate world offer valuable insight into Army logistics challenges. Two key concepts will be offered here. First, enabled by transportation planning and materiel management, "logistics" has traditionally been defined as the "forward flow" of materials from suppliers through a series of production and distribution stages to customers. More recently, "supply chain" design concepts and management theory expanded to incorporate two other "flows" through these logistics stages as well: demand information and cash flows "back" through the structure. This view of multiple, interacting flow "templates" (Figure 4) then led to the second concept, now known as the "bullwhip" effect, which illuminates the enormous consequences of these independently operating stages and interacting templates on the system. Actual consumer demand is magnified at successive stages as a consequence of incomplete and delayed information rippling back through the supply chain, thereby causing amplification, oscillation and time lags of the original demand signal. This induced, cascading variability, which necessitates greater inventory levels to accommodate such volatility, has recently been quantified in the academic literature and grows geometrically as the number of independent "stages" in the supply chain increases. The value of information sharing and collaboration across the stages, in contrast to independently operating stages, has also been quantified and shown to dramatically reduce system variability and associated requirements for buffer inventory while actually improving performance (e.g., reduced "stockouts"). In essence, shared information and collaboration has the effect of "collapsing" several independent stages into only a few "virtual" stages. (See Figure 5.) In practice, one example of implementing supply chain collaboration is referred to as "vendor managed inventory."

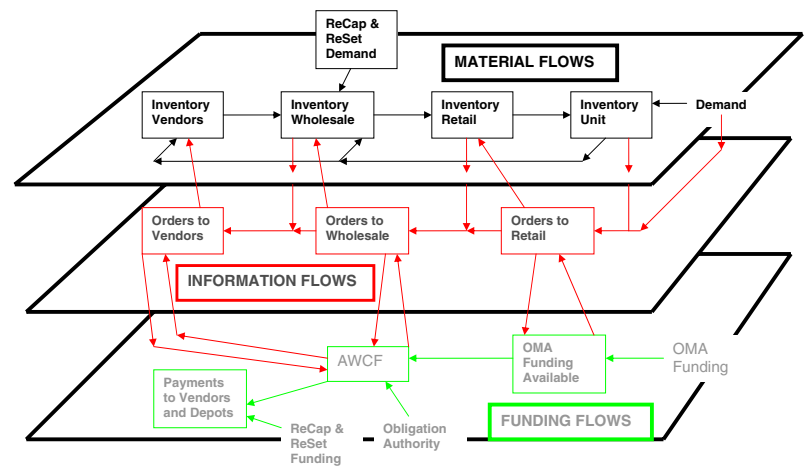

Figure 4: Three-Dimensional Nature of Supply Chains 


\section{Multi-stage Systems: $\operatorname{Var}\left(q^{k}\right) / \operatorname{Var}(D)$}

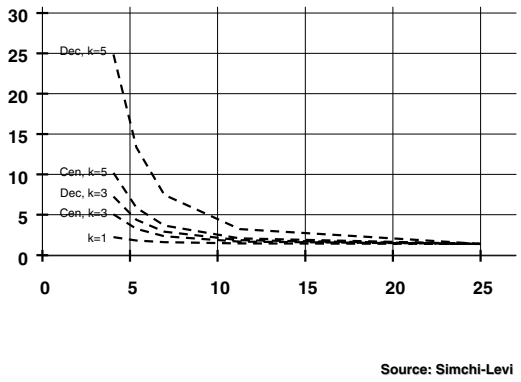

Figure 5: Effect of Shared Information

We have recently been able to clearly demonstrate that the existing aviation logistics structure is indeed vulnerable to the "bullwhip" (Killingsworth 2004). This ability, using system dynamics modeling, provides significant explanatory power regarding the "death spiral" conditions characterizing the current environment mentioned above. Our analytical challenge now is to better understand, then attack each of the root causes that contribute to this variability both within each stage and across the system of stages. The stages can then be "linked" together using optimization techniques including, for example, Readiness Based Sparing (RBS) which is a marginal analysis approach to optimize retail stock to desired readiness at the unit stage. By reducing uncertainty and improving efficiency within each stage, logistics system performance is moving toward an efficient frontier in the cost-availability trade space. (See Figure 6.) Major efficiency gain initiatives we are attempting to quantify through analytical demonstrations, field experiments and testing include: development of a "readiness equation" for the unit stage, implementing RBS and centralized risk pooling in the retail stage; using empirically-derived, mission-based forecasts for the demand stage (our anticipated subject for the next article); and reducing the delay in the retrograde stage. Then, working "backwards" through the supply chain from the point of readiness production, the stages can be linked together, conceptually using a dynamic programming application, where the links between stages are optimized, enabling "resources-to-readiness" investment decisions to be made with dramatically improved accuracy (Goldberg and Kimko 2003). Return on investment estimates can also then be reliably performed for the variety of initiatives that have been proposed and cost- benefit assessments made that would enable this "analytical architecture" to guide an ambitious Logistics Transformation endeavor, consistent with a strategic plan for implementation, by "pushing the envelope" of continuous improvement. (See Figure 7.)

\section{Readiness Based Sparing at 101st Airborne - Blackhawk Parts}

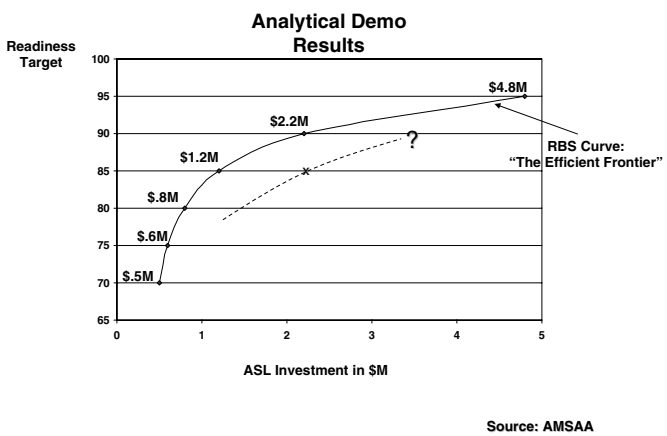

Figure 6: Improving Logistics System Performance

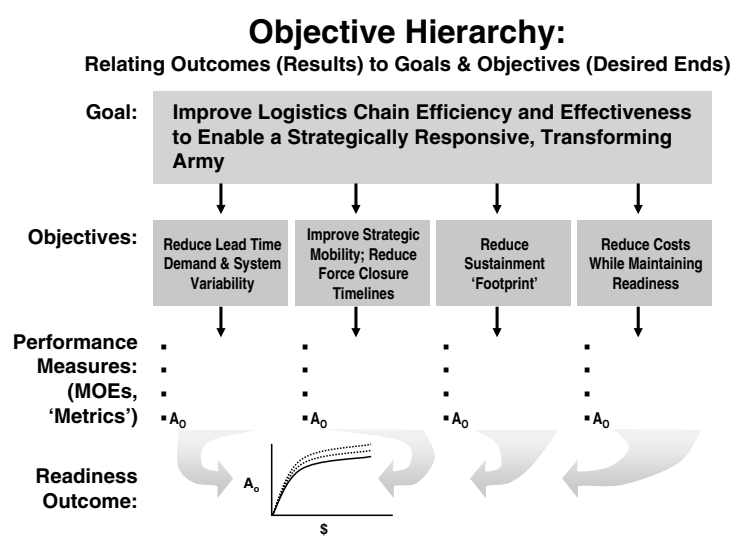

Figure 7: Implementing the Logistics Transformation

\section{OBSERVATIONS}

These improvements to our "business processes" are necessary to fully capitalize upon the enterprise-wide promise offered by "information technology." Enormous sums have been invested by the corporate world in IT-based enterprise resource planning "solutions" with very mixed results (Brown 2003). The emerging evidence suggests that dramatic improvements in performance and competitiveness can be achieved, but this success has been limited to those organizations that have applied IT to an existing foundation of mature, efficient and appropriate business processes. IT cannot substitute for lack of such a foundation and, in fact, the evidence suggests that such attempts not only fail to achieve any performance increase-despite large and lengthy investment efforts-but actually result in reduced performance (Heinrich and Simchi-Levi 2004).

Additionally, the intent is certainly NOT to blindly adopt the latest management "fad" inundating the corporate world but rather to consider adapting proven concepts to 
the unique needs and challenges the Army now faces. For example, Lean manufacturing concepts have helped firms to become more competitive through the application of "justin-time" principles which exchange "industrial age" mass for "information age" velocity. However, "just-in-time" manufacturing concepts, though a powerful inventory reduction method, need stable, predictable, "linear" supply chains for maximum efficiency. Even when enabled by IT, such supply chains are vulnerable, fragile and easily disrupted. A more appropriate analogy for Army logistics is a flexible, robust logistics "network"; not a hierarchical or serial "chain" but rather a network "web"-as in spider web-which is then enabled by both a strong analytical foundation and information technology to achieve an integrated, flexible and efficient logistics capability.

Finally, an honest, forthright post-mortem begs the question of how the "state of Army logistics" has become what it now is. In retrospect, the drawdown during the decade of the '90s completely gutted the analytical "braintrust" of logistics-focused, military operations research/systems analysts within Army Materiel Command: officer ORSA authorizations (FA 49) declined from 55 in FY89, including 5 colonels, to 0 by FY00 and have remained at 0 since then; Army Civilians (GS 1515) also took a disproportionate share of cuts as well declining from almost half of Army- wide authorizations in FY90 to less than a third by FY02; many of those that remain, however, are providing customer "matrix support" to Program Management Offices as cost analysts. In the case of AMCOM, no budget to support "outsourcing" logistics systems analysis, research and studies had existed for over five years until this project was initiated a few months ago. The Army Logistics Community must organize for success starting with an investment in analytical "recon."

\section{FINAL THOUGHTS}

Beyond the obvious opportunity to inform others about this study effort, we also hope this project will serve as a catalyst for an intellectual and professional resurgence in military logistics systems analysis. We wish to engage the larger military operations research and logistics communities and encourage your participation to collectively pursue this challenge.

\section{REFERENCES}

Association of the United States Army. 2004. The new paradigm: Bringing U.S. Army logistics in to the 21st century [online]. Association of the United States Army, Arlington, Virginia. Available online via $<w w w$. ausa. org/ilw> [accessed August 11, 2004].

Brown, G. G. 2003. Has IT made OR obsolete? Paper presented at the INFORMS Conference on OR/MS Practice, Phoenix, Arizona.
Goldberg, L., and D. Kimko. 2003. An enlistment early warning system to prevent the next recruiting crises. Report D-2720, Institute for Defense Analyses, Alexandria, Virginia.

Headquarters Department of the Army. 2003. Army logistics transformation: Management, synchronization and integration to achieve full spectrum capabilities. Office of the Deputy Chief of Staff, U.S. Army, Washington, D.C.

Heinrich, C. E., and D. Simchi-Levi. 2004. Is there a link between IT investment and financial performance? Draft Report, MIT Executive Series on Management and Technology: Supply Chain Strategy and Management, Massachusetts Institute of Technology, Cambridge, Masssachusetts.

Kallock, R. W., and L. R. Williams. 2004. From factory to foxhole. Supply Chain Management Review 8 (4): $46-53$.

Killingsworth, W. R. 2004. An integrated analysis of the supply chain for Army aviation spares. Briefing, Office of Economic Development, University of Alabama at Huntsville, Huntsville, Alabama.

Parlier, G. 2003. Enabling a strategically responsive, transforming Army: A systems approach to improve logistics chain efficiency and effectiveness. Briefing, U.S. Army Aviation and Missile Command, Redstone Arsenal, Alabama.

\section{AUTHOR BIOGRAPHY}

GREG PARLIER is a graduate of the United States Military Academy at West Point. He earned an MS in ORSA Engineering from the Naval Postgraduate School in 1983, an MA in National Security Studies from the Walsh School of Foreign Service at Georgetown University in 1988, and is working toward completion of a $\mathrm{PhD}$ in Systems Engineering initiated at the University of Virginia. Currently he is a Senior Research Information Scientist in the Office of Economic Development of the University of Alabama in Huntsville. 